\title{
Research on the application of automatic goods sorting system Qi Li ${ }^{1, a}$, Xiaohong Cheng ${ }^{2, b}$ \\ ${ }^{1}$ Beijing Information Technology College,China, Beijing, 100015, China \\ ${ }^{2}$ Beijing Wuzi University Beijing ,China, Beijing, 101149, China \\ aemail: liq@bitc.edu.cn, bemail:bj201801@163.com
}

Keywords: PLC controller; Sensor; Pneumatic control; Material sorting

\begin{abstract}
Sorting control system plays a very important role in the advanced manufacturing field. Is an important part of modern logistics and industrial control system, implementation of materials at the same time sorting over continuous multilayer. In various industries such as: logistics and distribution center, post office, warehouse and other industries have been widely used. With the development of science and technology, the sorting system has begun to use a variety of automatic equipment, information technology and computer control technology has become an important means of processing and information transmission. Although there are some parts of the sorting system in the process of the need to have artificial participation, but the intensity of the operation is getting smaller and smaller. The automatic sorting system is also made with the mechanical completion of the sorting operation. The main characteristics and the development trend of the modern sorting system is the automation, mechanization and intelligence.

This paper mainly focuses on the application of PLC in the cargo sorting system, adopting the integrated design idea, taking PLC as the main controller, and designed a set of automatic sorting device with low cost and high efficiency. Combined with sensing technology, pneumatic device, network technology, frequency conversion technology, position control and other technology to achieve automatic control products. The system has the characteristics of high degree of automation, high precision, stable operation and easy control, and can realize the control system of different goods into different positions.
\end{abstract}

\section{Introduction}

Automatic sorting system in the distribution center is a very necessary position. With very high efficiency, generally speaking, the number of goods per hour is up to 6000-12000 box, it can be said that automatic sorting is one of the important factors to improve the logistics distribution and transmission efficiency. After World War II, in many countries, such as Japan, Japan and other countries in the logistics center is widely used by a automatic sorting system, at present, the system has been widely used in the development of large and medium-sized logistics center.

Automatic sorting system operation process, the logistics center to absorb a variety of products, and in the shortest time to unload, and then according to the type of goods, suppliers and send location to the specified destination, with the same time, if the supplier to inform the logistics center according to their own requirements of the delivery of goods, sorting system can be in the shortest time, from the top shelf storage system to accurately find the goods and their location, and by the need to. From different storage position out of a different number of goods according to the delivery locations of different kinds of transportation to different tally area or distribution platform concentrated, to casual delivery vehicles.

A, can be continuous, large quantities of goods classification. Due to the use of automatic production lines, automatic sorting system is not subject to climate, time, physical conditions, such as continuous operation, and due to the number of automatic sorting system unit time sorting, sorting ability sorting system can continuously run for more than 100 hours, 7000 pieces per hour classification, such as the use of manual sorting 150 pieces / hour or so, while sorting workers can not work 8 hours continuously in this labor intensity.

$\mathrm{B}$, the classification error rate is very low. The classification error of automatic classification 
system depends mainly on the accuracy of the information input, which in turn depends on the classification of information input system, if the use of manual keyboard input or speech recognition method, the error rate of more than 3\%, such as the use of bar code scanning input, unless the bar code printing itself is wrong, or you will not go wrong. Therefore, the automatic sorting system mainly uses the bar code technology to identify the goods. 3 , the basic realization of the one of the foreign automatic sorting system is to reduce the use of personnel, reduce the labor intensity of workers, improve the use efficiency of the staff, so automatic sorting system can reduce the use of the basic is unmanned.

But the automatic sorting system is also very high, and the automatic sorting system is suitable for the ordered goods at the bottom of flat and rigid packaging. Packaging, soft and uneven bottom of the packaging, packaging, easy deformation, easy damage, long, slim, overweight, ultra high, can not overturn the commodity can not be used to sort the automatic classification machine, so in order to make most of the goods have been completed mechanical automatic sorting, can take two measures: first, to promote the standardization of packaging, so that most of the commodity packaging is in line with national standards; two is based on the sorting of most of the commodity packaging function custom specific classification machine. But it is very difficult for all suppliers to meet the national packaging, the custom characteristic classification machine, so that the hardware cost of the rise, and it is more general than the general. Therefore, the company should be based on the operation and the packaging of the goods decided to establish or set up what kind of automatic sorting system.

\section{Control requirements for sorting system}

This paper is material sorting system based on the distinction between different materials and design, mainly to achieve the automatic sorting of the material of iron, aluminum and different colors, and the specific control process:

(a)On the power supply, press the start switch SB1, the system into the starting state, the light green L2.

(b)After the system is started, the lower material sensor SQ1 (photoelectric sensor device) detects the material tank without material or the cylinder is not reset, the conveyor belt must continue to run a stroke $10 \mathrm{~S}$ automatically stop, the indicator light red L1 light.

(c) After the system is started, the lower material sensor (photoelectric sensing device) is detected by the material, the material feeding cylinder every $2 \mathrm{~S}$ action once, the action time is maintained for $1 \mathrm{~S}$, the material is pushed to the conveyor belt, the object to be measured is running on the conveyor belt, and counting.

(d)When the inductance sensor SA detects the iron material, and its corresponding proximity switch SQ2 sensing the material close to the material, the iron out of the cylinder will be pushed down, and count.

(e) When the capacitance sensor detect SB to the material for aluminum and its corresponding close to switch SQ3 induction material close to, aluminum cylinder action will be detected material and the count.

(f) When the color detection sensor SC detects the material is red, and its corresponding proximity switch SQ4 sensing material is close to the material, the red discharge cylinder moves the object to be measured, and then counts the.

(g)The remaining material on the conveyor belt continue to transmit, when finally corresponding to the chute proximity switch induction material close to the material feeding cylinder action will be detected push, and the count.

The sorting control system is composed of the program flow chart of the control system, which is shown in Figure 1. 


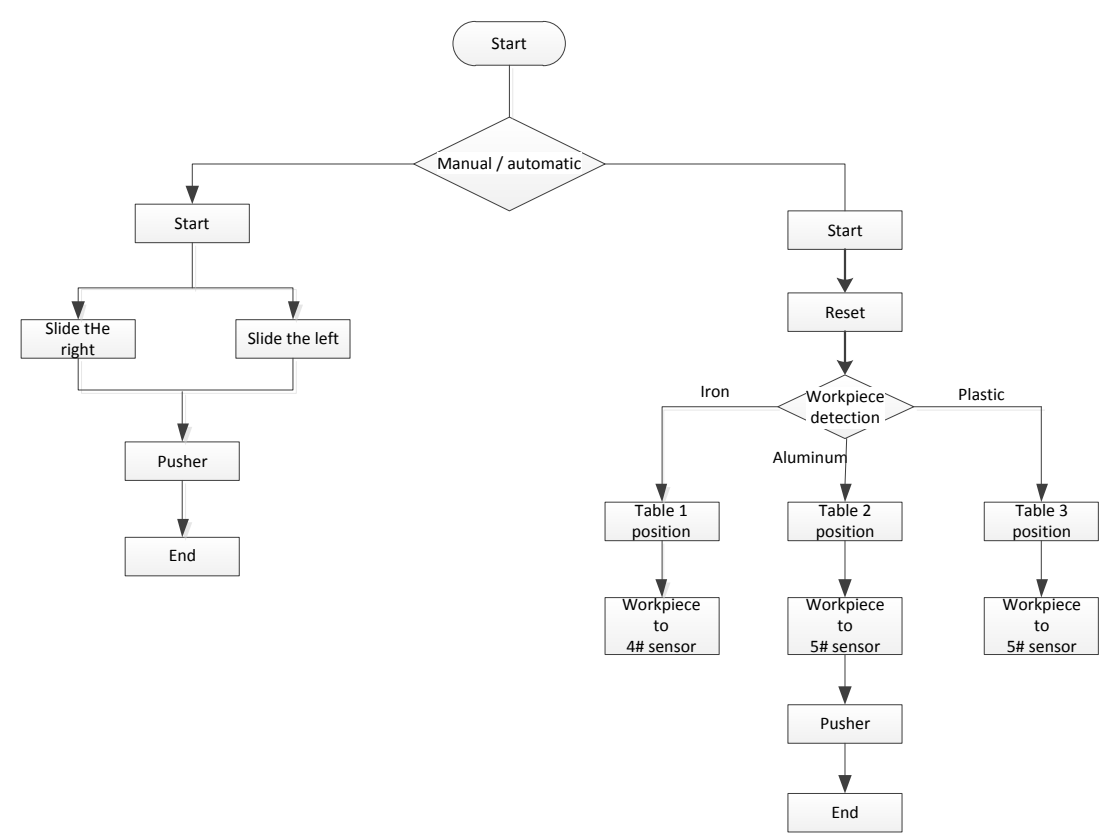

Figure 1 System control flow chart

\section{Hardware design of sorting system}

According to the physical structure, the PLC can be divided into integral and modular, and the average price of each I / O point of integrated PLC is cheaper, generally using PLC module type, in the small control system. But convenient and flexible, modular PLC features I / O point, such as the ratio of the number of output, input point and point, and type of I / O module, the use of special I / $\mathrm{O}$ module is much greater than the integral PLC, to determine the scope of the fault in the maintenance module, it is also very convenient, and therefore more complex, requiring higher general choice of modular PLC system. Considering the control of the system is relatively simple, it is suitable for the selection of the structure of the whole PLC.

At present, for the color recognition sensor has two basic types: (1) color code sensor, which uses an incandescent light source or monochromatic LED light source; (2) RGB (red, green, and blue) color sensor, object detection of reflectance of three primary colors to identify the body color. Many of these devices are warm reflective, beam type, fiber type, packaged in a variety of metal and polycarbonate housing, the typical output is: NPN type and PNP type, relay.

In order to facilitate the preparation of PCL control program, the economic benefits of the company and its various conditions, in this design, the choice of RGB color sensor as a device to identify the color of the material, relay output mode, making the PLC control system simple, more easy to achieve control system.

\section{Analysis of driving part}

The system is driven by the drive device, which is divided into pneumatic, hydraulic, electrical, electrical, mechanical, hydraulic and electrical combination and so on. At present, there are mainly three kinds of driving modes, which are air pressure, hydraulic pressure and electric power.

Pneumatic drive, low cost, simple structure, high power volume ratio, no pollution and strong anti-interference, in the hands of industrial machinery applications more. On the other hand, pneumatic technology is a kind of "cheap automation technology", because of the continuous improvement of the performance of components, reduce production costs, widely used in modern industrial production areas. In modern equipment and automated production lines, almost all equipped with pneumatic systems. Pneumatic manipulator technology has become an important tool to meet the requirements of many industry production practices.

Hydraulic drive, power weight ratio is large, can achieve frequent and smooth transmission and commutation, easy to achieve overload protection, can be lubricated, long service life. But also exist 
in the oil is easy to pollute the environment, need to be equipped with oil source, the cost is higher, the work noise.

Electric drive, high control accuracy, high driving force, fast response, signal detection, transmission, processing. But the electric drive mode is expensive, which limits the application of electric drive in some situations. Therefore, people are looking for other ways of economic application.

\section{Conclusion}

In this paper, the working principle and application fields of PLC and configuration technology are introduced, and the principle of automatic and sorting system is introduced. The design idea of the whole system is considered, and a material conveying and sorting system is designed. This system with the PLC as the main controller, combined with the technology of sensor, pneumatic device and position control technology, and the use of ladder diagram programming, realize the automatic sorting of the material of iron, aluminum and different colors. With the characteristics of high degree of automation, easy control, stable operation, high sorting accuracy, the different sorting objects, a little modification of the system to achieve the requirements. According to the actual needs of material sorting using PLC design of the material sorting system.

\section{References}

[1]She Yong. The design and implementation of intelligent electronic label goods sorting system[J]. Logistics technology, 2014,(11)

[2]Xiao Longxiang. The application of the block sorting system in express delivery company [J]. logistics technology (equipment version), 2014, (7): 86-89.

[3]Ma Yanling, Li Zhe.PLC application in automatic sorting of goods [J]. Inner Mongolia technology and economy, 2006, (10): 58-59.

[4]Zhu Jianhong, Wu Xiao, Based on S7-300 logistics control system design [J]. manufacturing automation, 2009,31 (2): 108-110123.

[5]Liu Yanxia, Lu Qian Ren Zi Hui. Based on Kingview and PLC goods automatic identification system [J]. Instrument technique and sensor, 2013, (6): 55-58. 